\title{
Switched flow systems: pseudo billiard dynamics
}

\author{
Michael Blank, Leonid Bunimovich ${ }^{\dagger}$
}

November 10, 2018

\begin{abstract}
We study a class of dynamical systems which generalizes and unifies some models arising in the analysis of switched flow systems in manufacturing. General properties of these dynamical systems, called pseudo billiards, as well as some their perturbations are discussed.
\end{abstract}

\section{Introduction}

In a series of papers dynamics of some basic models in manufacturing, called switched arrival and switched server systems, has been studied (Chase, Serrano and Ramadge 1993, Horn and Ramadge 1997, Katzorke and Pikovsky 2000, Peters and Parlitz 2003, Schurmann and Hoffman 1995). These models describe the local blocks of many systems in manufacturing and logistics, where one production unit (e.g. a work station) has to load a number of subsequent production units or one production unit has to unload (serve) some foregoing production unit. Here we introduce a new class of dynamical systems, called pseudo billiards, which contains switched arrival and switched server systems as well as some natural generalizations of these models. Dynamical and statistical properties of pseudo billiards and their stability against random and some deterministic perturbations are studied. It is shown that pseudo billiards can demonstrate chaotic, stable and neutral behavior. Sufficient conditions for each type of behavior and examples with their coexistence are given. The dynamics of the switched arrival system is considered in Subsection 2.1 and the switched server system in Subsection 2.3 respectively. Some perturbations of these models are discussed in Subsection 2.2 and 2.3.

\section{Mathematical background}

\subsection{Standard model}

Consider a system consisting of $N$ buffers, and one server (see Fig. 1). We refer to the content of a buffer as "material", and depending on the setup the material will be considered as a continuous object (e.g., fluid) or a discrete one (e.g., packets). Material is removed from buffer $i$ at a fixed rate $\rho_{i}>0\left(\sum_{i}^{N} \rho_{i}=1\right)$. On the other hand, the server delivers material to a selected buffer at unit rate, and the selection is done as follows: a threshold $\nu_{i} \geq 0$ is assigned to each buffer and the server switches instantaneously to the buffer in which the level of material falls below the assigned threshold. Otherwise the switch between the buffers happen only after the complete work on the current buffer is finished. If two or several buffers become empty at the same moment of time then server chooses a buffer according to some (cyclic) order. To avoid overflow or complete draining of all buffers a balance condition is necessary: the rate of filling is equal to $\sum \rho_{i}$.

The state of this system is completely described by the $N$-dimensional vector $x \in \mathbb{R}^{N}$ whose $i$-th coordinate $x_{i}$ represents the amount of material in the $i$-th buffer and the number of the buffer serving at the moment. Thus the evolution can be described as follows. The current state (which we associate to a point in $\left.\mathbb{R}^{N}\right)$ moves uniformly and linearly inside the bounded region $\left\{x \in \mathbb{R}^{N}: \sum_{i} x_{i}=\right.$ $\left.\sum_{i} \rho_{i}, \nu_{i} \leq x_{i} \leq \bar{\nu}_{i}\right\}$, i.e.

$$
\dot{x}(t):=e^{(i)}-\rho,
$$

${ }^{*}$ Russian Academy of Sciences, and Georgia Institute of Technology; e-mail: blank@iitp.ru

${ }^{\dagger}$ School of Mathematics, Georgia Institute of Technology; e-mail: bunimovh@math.gatech.edu 


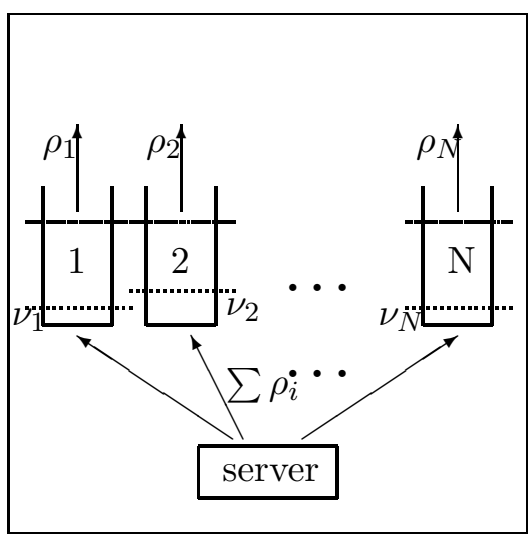

(a)

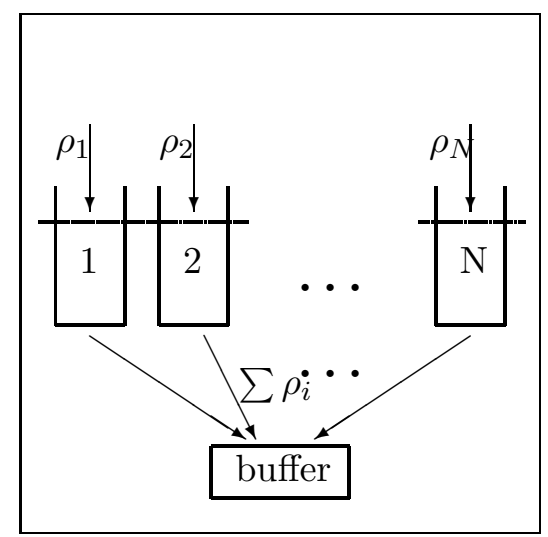

(b)

Figure 1: (a) Switched arrival system; (b) switched server system

where $e^{(i)}$ is the $i$-th canonical unit vector in $\mathbb{R}^{N}$ and $\rho:=\left(\rho_{1}, \ldots, \rho_{N}\right) \in \mathbb{R}^{N}$. After a collision with the boundary of this region, i.e. when one of the coordinates, say $x_{j}$, becomes equal to $\nu_{j}$, the velocity changes instantaneously to a new vector $e^{(j)}-\rho$ which depends only on the number of the buffer $j$ which will be served.

In the trivial cases $N=1$ or $N=2$ the dynamics is, obviously, periodic. In the simplest nontrivial case, when $N=3, \rho_{i}=1 / 3$, and $\nu_{i}=0$ the dynamics of this system is equivalent to the motion of a particle in the equilateral unit triangle (see Fig. 2(a)) which moves with the constant velocity inside the triangle and changes it instantaneously at the moment of a collision with the boundary to the velocity perpendicular to the corresponding side of the triangle. The motion inside the triangle corresponds to the situation when the server delivers material to the selected buffer, while switching between different buffers is described by the collision rule. Due to the symmetry the direction of the velocity after the collision is perpendicular to the side of the triangle where the collision took place.

Due to similarity of this dynamics to the motion of a ball in a billiard game, we call systems of this type pseudo billiards (in the literature one can find also the notion "strange billiard" used to describe a special particular case of pseudo billiards, see, e.g. (Peters and Parlitz 2003, Schurmann and Hoffman 1995)).

To be precise, by the pseudo billiard we shall mean dynamics of the particle which moves with the constant velocity inside a given region (not necessarily a polyhedron) and changes it instantaneously at the moment of a collision with the boundary to the velocity defined by a given vector field (not necessarily a constant one) on the boundary of the region. Note that a similar situation arises for billiards in a strong magnetic or in the gravitational field, where only the angle with the field matters.

A straightforward generalization of the considered above case of three equal buffers to the general system of buffers with $N \geq 3, \rho_{i}=1 / N$, and $\nu_{i}=0$ leads to the motion of the particle with unit velocity in the equilateral unit $(N-1)$-dimensional pyramid up to a moment of a collision with some $(N-2)$-dimensional face of the pyramid when the direction of the velocity changes instantaneously to the one perpendicular to this face.

More general model with arbitrary rates $\rho_{i}>0$ and small enough thresholds $\nu_{i} \geq 0$ leads to a similar picture, where the $i$-th vertex of the equilateral unit $(N-1)$-dimensional pyramid is "cut" by the hyperplanes perpendicular to the corresponding axes at the level $\nu_{i}$ and the "perpendicular reflection law" is changed to the law defined by the relation between $\rho_{i}, 1 \leq i \leq N$ in the case of the reflection law from the 'main' part of the face, while the reflection from the "cut" part is inherited from the corresponding 'normal' face (see details in Section 2.2.).

Therefore all these situations can be considered as special cases of the motion of the particle with unit velocity in a polyhedron $\mathcal{X} \in \mathbb{R}^{d}$ with the "reflection law" on boundaries defined by a given vector field. We will refer to the simplest situation when $\mathcal{X} \in \mathbb{R}^{d}$ is the equilateral unit $d$-dimensional pyramid and the restriction of the vector field to each of its $(d-1)$-dimensional faces is a constant vector-field as to the standard model. 


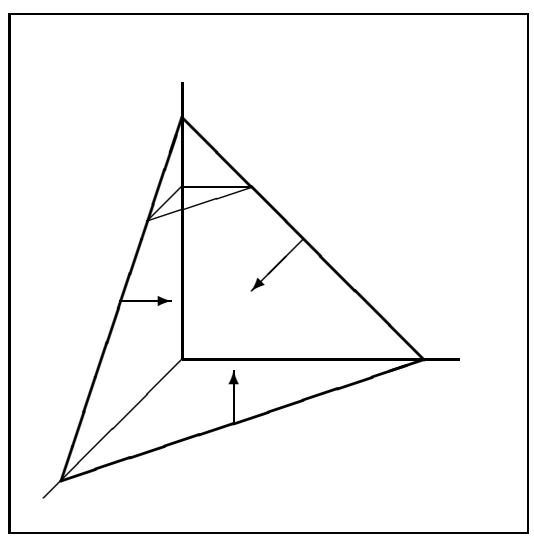

(a)

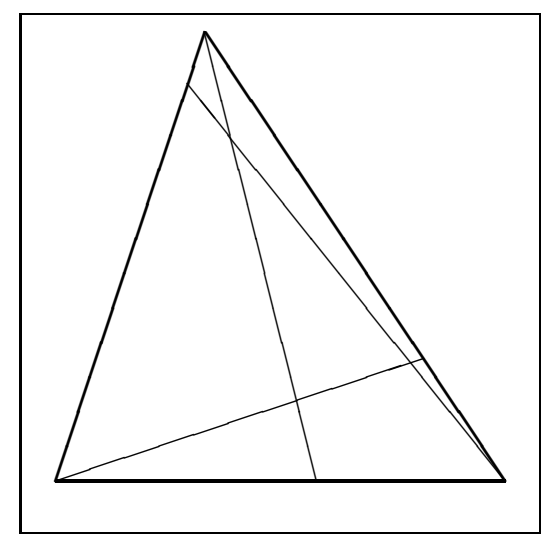

(b)

Figure 2: (a) Pseudo billiard; (b) conditions of chaoticity

Denote by $X$ the union of $(d-1)$-dimensional faces of $\mathcal{X}$ and by $\Sigma$ the union of its $(d-2)$ dimensional faces. Note that the set $\Sigma$ has zero $(d-1)$-dimensional Lebesgue measure. The first return (Poincare) map from the set $X$ into itself we denote by $T$. Recall that for any point $x \in X$ its image $T x$ is defined as the next intersection of the trajectory of the point $x$ with $X$.

There are two major approaches to a rigorous analysis of dynamical systems of this type. One is based on construction of the so called Markov partition and study of the corresponding symbolic dynamics. The second, operator approach, deals with the action of the dynamical system in the space of measures. Both of these approaches were already used for the analysis of the standard model (at least of its 2-dimensional version, see, e.g., (Chase, Serrano and Ramadge 1993, Katzorke and Pikovsky 2000)), and we will start with general discussion of their advantages and restrictions.

Consider first a Markov partition. Observe that in the simplest case $\nu_{i}=0$ the dynamical system $(T, X)$ has the property that each $(d-1)$-dimensional face is mapped onto the union of some other $(d-1)$-dimensional faces and this mapping is one-to-one. A slight generalization of this property leads to partition of the phase space $X$ into a collection of connected components $X_{i}$ with piecewise smooth boundaries satisfying the following conditions:

- $\cup_{i} X_{i}=X$,

- $\operatorname{Int}\left(X_{i}\right) \cap \operatorname{Int}\left(X_{j}\right)=\emptyset \quad \forall i \neq j$,

- $T X_{i}=\cup_{j \in I_{i}} X_{j}$

- $\left.T\right|_{X_{i}}$ is a homeomorphism for each $i$,

which we shall call the strong Markov property, and the corresponding partitions will be called strong Markov partitions. The word 'strong' is used here to underline the distinction to the usage of Markov partitions in the case of hyperbolic systems, where only "stable" boundaries of elements of the partition are preserved.

Consider the action of the map $T$ in the space $\mathcal{M}(X)$ of probability measures on $X$. Recall that a measure $\mu \in \mathcal{M}(X)$ is called $T$-invariant if for any measurable set $A \subseteq X$ we have $\mu(A)=\mu\left(T^{-1} A\right)$, and ergodic with respect to $T$ if $\mu$-measure of any $T$-invariant set is equal either to 0 or to 1 . A dynamical system $(T, X)$ with a nonergodic invariant measure $\mu$ can be uniquely (up to sets of $\mu$ measure zero) decomposed into ergodic components, i.e. such subsets $\left\{Y_{i}\right\}$ that for each $i$ the restriction of the measure $\mu$ to $Y_{i}$ is an ergodic invariant measure for the restriction of the map $T$ to $Y_{i}$.

Let us fix a reference measure $m$ on $X$. Since in the examples considered in the paper the phase space typically will be a subset of the $d$-dimensional Euclidean space $\mathbb{R}^{d}$ and only Lebesgue reference measures will be considered. We shall say that a dynamical system $(T, X)$ is strongly chaotic if there is a $T$-invariant measure $\mu$ absolutely continuous with respect to the reference measure $m$ (i.e. $\mu$ has a density with respect to $m$ ). 
The simplest maps having this property are the so called expanding maps. A map $T$ is expanding if its Jacobian $\left.D T\right|_{x}$ is an expanding matrix at any point $x \in X$. Recall that a matrix $A$ is called expanding if there is a number $\lambda_{A}>1$ called expanding constant such that for any vector $z$ we have $|A z| \geq \lambda_{A}|z|$, where $|z|$ is the Euclidean norm of the vector $z$.

Theorem 2.1 Let $T: X \rightarrow X$ be a piecewise $C^{1}$ map having a finite strong Markov partition $\left\{X_{i}\right\}_{i=1}^{K}$. Then the phase space of the dynamical system $(T, X)$ can be decomposed into at most $K$ ergodic components and in each ergodic component all Lyapunov exponents are either all positive or all equal zero. If $d=2$ and the map $T$ is continuous, then the system is either ergodic and strongly chaotic (has an a.c.i.m.), or pure neutral (each trajectory is eventually periodic).

Proof. Elements of the Markov partition $\left\{X_{i}\right\}$ can be divided into groups (components of transitivity) with respect to the possibility to go from one of them to another under the action of $T$. Namely two elements $X_{i}, X_{j}$ of the Markov partition belong to the same component of transitivity if there is a positive integer $n$ such that the interior part $\operatorname{Int}\left(T^{n} X_{i} \cap X_{j}\right) \neq \emptyset$.

Consider a sequence of matrices $A_{n}:=\prod_{k=0}^{n-1} D T\left(T^{k} x\right)$, where $D T(y)$ is the Jacobian of the map $T$ at point $y$. According to Multiplicative Ergodic Theorem (see, e.g., (Katok and Hasselblatt 1995)) for a.a. $x \in X$ the limits as $n \rightarrow \infty$ of logarithms of eigenvalues of the matrix $A_{n}$ divided by $n$ (called Lyapunov exponents of the map $T$ ) exist and do not depend on $x$ (on each component of transitivity). Observe now that due to the assumption on existence of a finite strong Markov partition all eigenvalues should be nonnegative (otherwise we will get the contraction, which contradicts the strong Markov property).

If some of the Lyapunov exponents are equal to 0 then the corresponding components of transitivity can be nonergodic (consider, for example, a rational rotation of the circle $T x=x+\alpha \quad(\bmod 2 \pi)$ where $\alpha / \pi$ is a rational number). On the other hand, on each ergodic component the system is either pure expanding, or pure neutral.

Consider now the case $d=2$ and assume that the only Lyapunov exponent of this system is positive on some component of transitivity. Since we have a finite Markov partition, there is a constant $n_{0}$ such that the map $T^{n_{0}}$ is strictly expanding. Clearly the map $T^{n_{0}}$ is still continuous and the strong Markov property holds for it with respect to the same Markov partition. Thus due to the continuity of the map $\forall i \quad T^{n_{0}} X_{i}$ is a union of neighboring elements of the Markov partition, and the Lebesgue measure of this union is strictly larger than the Lebesgue measure of the set $X_{i}$. Therefore there is another constant $n_{1}$ such $\forall i \quad T^{n_{0} \cdot n_{1}} X_{i}=X$. Thus the map $T^{n_{0} \cdot n_{1}}$ is uniformly expanding and has an absolutely continuous (with respect to Lebesgue measure) invariant measure.

If the dimension $d>2$ the situation can be more complex and a chaotic regime may coexist with a neutral periodic one. Moreover if we drop the assumption of the continuity of the map $T$ the coexistence may take place even if $d=2$ and a map is piecewise linear (see below). In the multidimensional case it is much easier to construct a counterexample of the absence of ergodicity of the system because one can consider a direct product of a chaotic 2-dimensional system and a neutral one, which is certainly nonergodic. On the other hand, it turned out to be rather difficult (but still possible) to construct a counterexample with a map having discontinuities only on the boundary of a strong Markov partitions and being non ergodic. The following result demonstrates coexistence of chaotic and neutral regimes in a 2-dimensional pseudo billiard.

Theorem 2.2 There exists a 2-dimensional pseudo billiard generated by a discontinuous map $T$ such that it has both strongly chaotic and neutral components of transitivity.

Proof. Consider the pseudo billiard depicted in Fig. 3. Here a chaotic regime (on the component AG,FG,FE,BC,CD) coexists with a neutral one (on another component AB,ED). In this construction we assume that the pairs of straight lines (AB, GD), (BG,CE), (AE,BD), and (AE,GF) are parallel to each other, and that $|G F|<|F E|,|C D|<|B C|<|G F|+|E F|$, and $|A G|<|B C|+|C D|$, which 

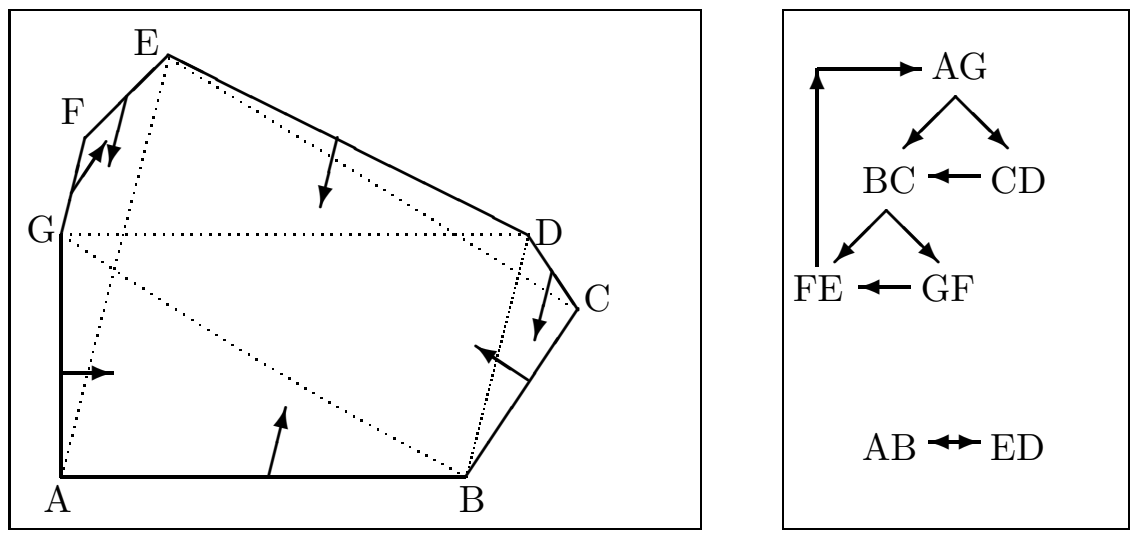

Figure 3: Coexistence of chaotic and neutral regimes

can be easily achieved. (Observe that we can weaken these assumptions by assuming that FE is mapped onto the union of AG and GF and the statement about the coexistence of two components still remains valid.) Under these assumptions the map is piecewise expanding on the components $\mathrm{AG}, \mathrm{FG}, \mathrm{FE}, \mathrm{BC}, \mathrm{CD}$, and it is neutral on the remaining part of the boundary. The invariance of both these components follows from the construction.

The main disadvantage of the approach based on Markov partitions is that the construction of a partition is rather unstable with respect to various perturbations (except perturbations adapted to dynamics in a sense that the Markov partition is preserved under the perturbation, which is a very rare situation) and that 'typically' a dynamical system does not have a finite Markov partition (especially a strong one). Moreover, even if a dynamical system has a finite Markov partition, then 'typically' under arbitrary small perturbations this property does not survive.

Consider the simplest case of the standard model with $N=3$ and a small 'cutting' of one of its faces. Here by the cutting operation we mean the partition of the original phase space in two parts by a hyperplane. Choosing one of those parts we get the phase space for the perturbed system, and by the amplitude of the perturbation (its smallness) we mean the volume of the additional part. It is straightforward to show that for almost all 'cuttings' this new system does not have a strong finite Markov partition. To deal with this situation we shall apply the operator approach.

Observe that in this case some iteration of the map $T$ restricted to $X$ is a piecewise expanding $(\mathrm{PE})$ map, which means that there is a collection of connected sets $X_{i}$ with piecewise smooth boundaries satisfying the properties:

- $\cup_{i} X_{i}=X$,

- $\operatorname{Int}\left(X_{i}\right) \cap \operatorname{Int}\left(X_{j}\right)=\emptyset \quad \forall i \neq j$,

- $\left.T\right|_{X_{i}}$ is a $C^{1}$-homeomorphism for each $i$,

- the Jacobian $D\left(\left.T\right|_{X_{i}}\right)$ is an expanding matrix for all $i$ and $x \in X_{i}$.

The operator approach is well developed for the case of piecewise expanding maps (see, e.g., (Blank 2001)). For instance, it can be shown that if a map is piecewise expanding, then it is strongly chaotic. Recall that a map $\phi: X \rightarrow X$ is called an absolutely continuous homeomorphism if it is one to one and for any set $A$ of Lebesgue zero measure its image $\phi(A)$ has also zero Lebesgue measure.

The following statement is one of the basic observations in the theory of piecewise expanding maps.

Theorem 2.3 If a continuous map $T$ is conjugated to a piecewise expanding map $\tilde{T}$ by an absolutely continuous homeomorphism $\phi$ (i.e. $\phi T=\tilde{T} \phi)$, then all Lyapunov exponents of the map $T$ are positive and $T$ has an absolutely continuous invariant measure $\mu_{T}$. 
Consider now the simplest standard model, i.e. the case when $\mathcal{X}$ is the equilateral unit pyramid with triangular $(d-1)$-dimensional faces and on each face the vector field is perpendicular to it.

Theorem 2.4 If $d>2$ then for the standard model, the first return map $T$ is not piecewise expanding. On the other hand, the $(d-2)$-th iteration of this map (i.e. $\left.T^{d-2}\right)$ is piecewise expanding, and thus this map is strongly chaotic.

Proof. Let $d=3$. Fix one of the $(d-1)$-dimensional faces of the pyramid and denote by $A, B, C$ its vertices and by $O$ the orthogonal projection of the opposite vertex to this face. Choose two points $D \in A O$ and $E \in B O$ such that the lines $A B$ and $D E$ be parallel to each other. Then the image of the interval $D E$ under the action of $T$ is again an interval of the same length parallel to $D E$. Therefore there is no expansion in this direction.

Observe that if $d=3$ then the described above neutral direction cannot be mapped again to the neutral direction. Therefore in the case $d=3$ the second iterate of our map becomes expanding.

If the dimension $d \geq 3$, on each of the $(d-1)$-dimensional faces one can construct a $(d-2)$ dimensional simplex such that under the action of $T$ it will be mapped isometrically to the $(d-2)$ dimensional simplex on another face of $\mathcal{X}$. However, similarly to the 2 -dimensional case, only a $(d-3)$ dimensional face of this image will be mapped isometrically under the action of $T^{2}$. Thus after each successive iteration the dimension of the isometrically mapping simplex is decreasing by 1 , which finishes the proof.

If the assumption that the set of end-points of boundary segments is mapped into itself is not satisfied, the system can demonstrate any type of behavior, starting from the chaotic one and up to the appearance of globally stable periodic trajectories as well as to their coexistence.

In this case one can expect a complex behavior even for the standard model. The following simple assertion describes necessary and sufficient conditions for the existence of stable and chaotic regimes for this model.

Theorem 2.5 Consider the standard model. If for each vertex of $\mathcal{X}$ the straight line drawn parallel to the constant vector-field corresponding to the opposite face intersects the face in its inner point (see Fig. Q(b)) then this pseudo billiard system is strongly chaotic. Otherwise a pseudo billiard can have a globally stable periodic trajectory.

Proof. It is straightforward to check that under the assumptions of this theorem the map $T$ satisfies the strong Markov property with the Markov partition consisting of pieces with piecewise linear boundaries of $(d-1)$ dimensional faces of $\mathcal{X}$. Indeed, if the straight line from a vertex $A$ drawn parallel to the constant vector-field corresponding to the opposite face intersects this face, then the image of this face coincides with the union of $(d-1)$ dimensional faces having a common vertex $A$. Thus, the preimages of the $(d-1)$ dimensional faces of $\mathcal{X}$ generate the strong Markov partition. Observe now that the map $T$ is continuous, linear on elements of the partition, and that some iterate of each element of the partition covers $\mathcal{X}$. Therefore there exists a positive integer $n_{*}$ such that the map $T^{n_{*}}$ is piecewise expanding and maps each element of its strong Markov partition onto the complete phase space, and thus the system is strongly chaotic.

To show that if our condition does not hold the system can have a regular dynamics, consider the 2-dimensional case and assume in the triangle $A B C$ the face $A B$ is mapped to $A C$, the face $A C$ is mapped to $A B$ and $B C$ is mapped to $A B \cup A C$. Observe that it occurs if the straight lines from vertices $B$ and $C$ drawn parallel to the constant vector-field corresponding to the opposite faces (namely $A C$ and $A B$ ) do not intersect them. It is easy to show that in this situation the point $A$ is a globally stable fixed point of the map $T$.

Remark 2.6 At the first sight it looks like that the dynamics of this type can be analyzed (at least in the 2-dimensional case) by making use of the idea that the map $T$ is topologically conjugated to 


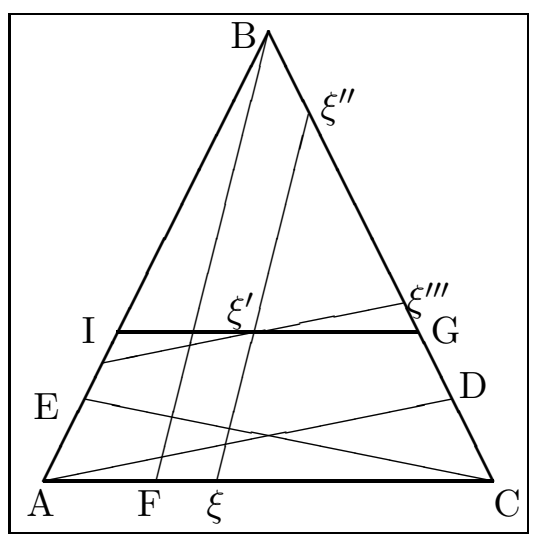

(a)

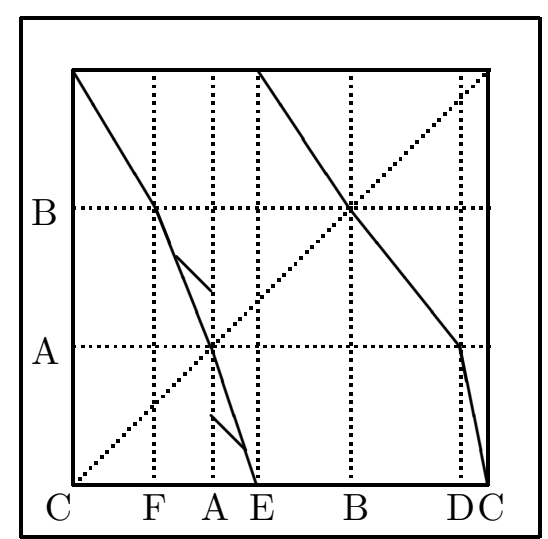

(b)

Figure 4: (a) Cutting perturbation, (b) modifications are shown near the point A

the binary one (i.e. to the map $x \rightarrow 2 x \quad(\bmod 1))$. Observe though that the topological conjugation is not enough to deduce any result about metric properties of the map (like the existence of a.c.i.m., etc.). In fact, one needs to have at least an absolutely continuous conjugation. Besides, even in the 2 -dimensional case this map is topologically conjugated to the map $x \rightarrow 1-\{2 x\}$ (where $\{\cdot\}$ stays for the fractional part of a number) rather than to the binary map. It is not known whether or not such a conjugation exists in the multidimensional case, which rules out a generalization of this approach to $N>3$ (recall that the dimension of the phase space of the first return map is $N-2$ ).

\subsection{Perturbations}

It is reasonable to consider the case with nonzero thresholds $\nu_{i}$ as a perturbation of the standard model (Peters and Parlitz 2003). We shall consider here three types of perturbations: "cutting" (describing nonzero thresholds), time discretization (corresponding to the case when all operations are performed in discrete time), and space discretization (when the work is a collection of discrete objects consisting, e.g., of finite size packets).

The "cutting" perturbation is defined by means of a collection of hyperplanes in $\mathbb{R}^{d}$ intersecting the polyhedron $\mathcal{X}$. A convex region obtained from $\mathcal{X}$ after the intersection defines the phase space of the perturbed system. The "cut" map is constructed as follows: a point moves along the corresponding vector-field until it reaches either the boundary of $\mathcal{X}$ or the "cut" piece. In the first case the map was already defined, while in the second case the vector-field (on the "cut" piece) is inherited from the point of the boundary of $\mathcal{X}$ to where the trajectory would come without the "cutting". Namely, one should prolong the trajectory up to the intersection with the boundary and the vector field at the intersection point defines the new vector-field (see Fig. 4). Let $\xi$ be a point on the boundary of $\mathcal{X}$ and let $\xi^{\prime}, \xi^{\prime \prime}$ be its images due to "cutting". Then the vector-field at the point $\xi^{\prime}$ is the same as the one at the point $\xi^{\prime \prime}$. Here by images of a point due to cutting we mean intersections of a trajectory started from this point with the old and new boundaries respectively. Observe that the image of the point $\xi$ is the point $\xi^{\prime \prime} \in X$, the line $\xi \xi^{\prime}$ is parallel to $B F$ and $\xi^{\prime} \xi^{\prime \prime \prime}$ is parallel to $A D$, while the directions $F B$ and $D A$ correspond to the restrictions of the vector-field to $A C$ and $B C$ respectively.

Theorem 2.7 Let the standard model $(T, \mathcal{X})$ satisfy the assumptions of Theorem 2.5. Suppose that the restriction of the vector-field to each $(d-1)$-dimensional face is orthogonal to it. Consider such "cutting" that under the action of $T$ each of the new $(d-1)$-dimensional faces obtained as a result of "cutting" is mapped to the same $(d-1)$-dimensional face as their ancestral faces do. Then the resulting system is strongly chaotic.

Proof. Observe that the "cut" system is again piecewise expanding and is piecewise linear on domains consisting of a finite number of simplexes. It is known (Tsujii 2001) that under these assumptions 


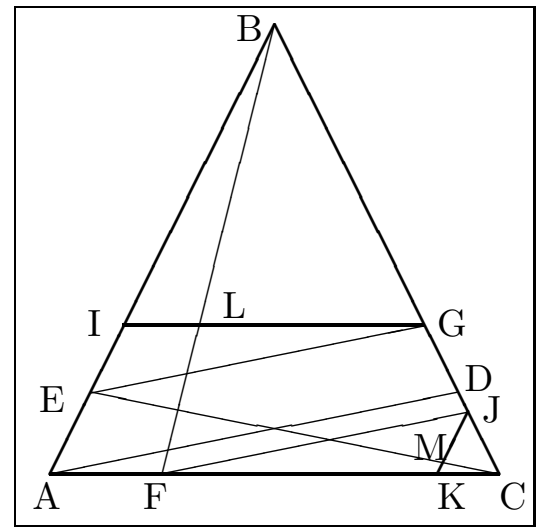

Figure 5: Existence of a stable periodic trajectory under "cutting"

the map has an absolutely continuous (with respect to Lebesgue measure) invariant measure, i.e. it is strongly chaotic.

Theorem 2.7 gives only sufficient conditions for the strong chaoticity. However they are close to the necessary ones. In particular, one cannot replace the assumption that the restriction of the vector-field to each $(d-1)$-dimensional face is orthogonal to it by the assumption that the map $T$ is piecewise expanding. The following example (see Fig. 5) shows that this cannot be done even if $d=2$.

Lemma 2.8 The map corresponding to the "cut" two-dimensional system depicted in Fig. 5 has a stable periodic trajectory.

Proof. It is easy to see that the system before "cuttings" was piecewise expanding. The lines $I G$ and $J K$ describe the "cuttings", which are constructed so that the conditions of Theorem [2.7] are satisfied. Denote the intersection points of straight lines $I G$ with $B F$ and $J K$ with $C F$ by $L$ and $M$ respectively. Then the segment $L G$ gets mapped under the action of $T$ to the segment $J M$, while the segment $J M$ gets mapped backward to the segment $L G$. Moreover, it is not hard to show that the absolute value of the derivative of the map along those two segments is strictly smaller than 1, which yields the contraction.

Remark 2.9 One can continue the construction in the proof above to get arbitrary small "cutting" which still leads to the existence of stable periodic trajectories. To do this let us draw a line from the point $I$ parallel to $A D$, whose intersection with $B C$ gives a new position of the "cutting" with the same properties.

The following Lemma gives geometric conditions equivalent to the assumptions of Theorem 2.7

Lemma 2.10 The following two conditions are equivalent:

- under the action of $T$ each $(d-1)$-dimensional face obtained by "cutting" gets mapped to the same $(d-1)$-dimensional face as its ancestral face;

- the "cut" piece intersects with only one of the straight lines, drawn from vertices parallel to the constant vector-fields corresponding to the opposite faces.

Proof. The proof is straightforward. (See Fig. 4(a).) 
In view of the billiard interpretation a time discretization of the dynamics means that inside the polyhedron $\mathcal{X}$ the particle moves in discrete steps with a given constant velocity. The only difference occurs near the boundary where in order not to go through the boundary (which is not permitted) the modulus of the velocity should be changed. Thus the Poincare section of the time discretized system at the boundary does not differ from the original one and therefore the invariant measure does not change under the time discretization.

The situation is different in case of the space discretization. For simplicity we shall consider only uniform space discretizations when all packets (describing the discretization) have the same size. In this case (again as in the previous situation) inside the polyhedron $\mathcal{X}$ the particle moves in discrete steps with the given constant velocity, whose modulus is equal to a size of the packet. This motion continues up to the moment when the distance to the boundary in some direction becomes smaller then the packets' size. Since this size cannot be made smaller, this moment has to be considered as a "collision" with the boundary and the velocity of the particle has to be changed to the one defined by the vector-field on this component of the boundary. Thus typically a trajectory of the particle never visits the boundary. Therefore the approach based on the Poincare section on the boundary does not make much sense. Numerical experiments (see, e.g., (Katzorke and Pikovsky 2000)) demonstrate very sensitive dependence of the statistical properties of the discretized system on the size of packets. To illustrate this consider the billiard type system in the square with the sides of length $\ell \in \mathbb{Z}_{+}$and with the unit vector-field on each side perpendicular to it. Clearly each trajectory of the unperturbed system is periodic with the time period $4 \ell$. Take now the uniform space discretization with the packet size $\varepsilon>0$. If $\varepsilon$ is a rational number, i.e. $\varepsilon=p / q$ where $p, q$ are positive integers then each trajectory of the discretized system starting from the boundary is periodic with the period $2 \ell q$. On the other hand, if $\varepsilon$ is irrational then each trajectory densely fills a certain region. Hence the system has an absolutely continuous invariant measure.

\subsection{Switched server system}

It makes sense to consider the situation opposite to the standard model (see Fig. 1(b)) when there is only one buffer and several servers (see, e.g., (Horn and Ramadge 1997) for technical details). A formal definition is as follows. Let $\mathcal{X} \in \mathbb{R}^{d}$ be a convex domain. Assume that $N$ vector fields are defined on the boundary $\partial \mathcal{X}$. One can think here about a polyhedron $\mathcal{X}$ with $d$-1-dimensional faces on each of which we fix $N$ different vector fields. We consider the dynamics of a particle moving with unit velocity inside $\mathcal{X}$ and changing the velocity at the moments of collision with the boundary $\partial \mathcal{X}$ to one of the vectors defined at the point of collision. A choice of one of $N$ possible vector fields is governed by a certain rule either deterministically (e.g., we enumerate the vector fields and each time after collision increase the number of the chosen field by one modulo $N$ ), or stochastically (e.g., we choose a vector field at random with respect to a certain probabilistic distribution $\left.p_{1}, \ldots, p_{N}\right)$. From the dynamical systems point of view this system can be represented as a skew product. Recall that the skew product of maps $F: X \rightarrow X$ and $G: X \times Y \rightarrow Y$ is defined as a new map $T: X \times Y \rightarrow X \times Y$ according to the formula $T(x, y):=(F(x), G(x, y))$. The first map $F$ is defined on the set of all integers and says which buffer to serve next time, while the second one $G$ is defined by the inverse branches of the map corresponding to the pseudo billiard system described in the previous section.

Indeed, in the simplest nontrivial case of three servers a Poincare section of the dynamics can be defined on the boundary of the equilateral triangle, on each side of which we consider two vector fields perpendicular to the remaining two sides of the triangle. Each trajectory of the system in this case coincides with one of the inverse branches of the dynamics of the model. Recall that the Poincare section of the standard model is described by a piecewise monotonic map with one-to-one monotonic branches.

Let $\mathcal{X}$ be the equilateral unit pyramid with $(d-1)$-dimensional faces on each of which we fix $N=d$ different constant vector fields. We assume similarly to Theorem 2.5 that for each vertex of $\mathcal{X}$ the straight lines drawn at this vertex parallel to each of vector fields, corresponding to the opposite $(d-1)$-dimensional faces, intersect with them at inner points. This assumption, which we shall call 
the standing assumption holds, e.g., if the vector fields are orthogonal to opposite faces.

Theorem 2.11 Consider an arbitrary deterministic setting. Assume that the standing assumption holds. Then any trajectory of this system converges exponentially fast to some stable periodic orbit. A number of such stable periodic orbits does not exceed $(d+1)^{d}$.

Proof. Observe that the standing assumption implies that for a given $(d-1)$-dimensional face and any possible choice of the constant vector field on this face the image of this face is a proper subset of one of the opposite faces. Therefore there exits a metrics on the boundary $\mathcal{X}$ for which the dynamics is uniformly contractive. The latter yields the needed statement.

Consider now a probabilistic version of this system, where a choice of the vector fields at a point $x \in \partial \mathcal{X}$ is governed by some probability distribution $p_{1}(x), \ldots, p_{N}(x)$, such that $\sum_{i=1}^{N} p_{i}(x)=1$ for each $x$. We assume that these distributions are strictly positive, i.e. $p_{i}(x)>q>0, i=1, \ldots, N$, and piecewise constant, i.e. there is a partition of the phase space such that $p_{i}(x)=$ const for all $i=1, \ldots, N$ and all $x$ belonging to the same element of the partition. This stochastic system is equivalent to the Markov chain defined as follows. Consider a collection of maps $\left\{T_{i}\right\}$ (which we call deterministic components) and collection of distributions $p_{1}(x), \ldots, p_{N}(x)$. Then the probability to go in one time step from a point $x \in X$ to a set $A \subset X$ is equal to $\sum_{i} p_{i}(x) \cdot 1_{T_{i} x \cap A}$, where $1_{B}$ is the characteristic function of the set $B$. Systems of this type are called iterated function system with probabilities (IFS).

Theorem 2.12 Suppose that the standing assumption holds. Then the Markov chain defined above has the unique invariant measure.

Proof. The Markov chain under study is ergodic due to positivity of transition probabilities. On the other hand, each deterministic component $T_{i}$ of this system is contractive (see the proof of Theorem 2.11), which yields the statement of Theorem using standard properties of contractive IFS (see, e.g. (Blank 2001[Theorem 4.2.1]) for details).

\section{Acknowledgements}

M.B. thanks Georgia Tech for a kind hospitality and his work was partially supported by the CRDF and RFBR grants. L.B. was partially supported by the NSF grant DMS - 0140165 and by the Humboldt Foundation. 


\section{References}

[1] Blank M., 2001, Stability and localization in chaotic dynamics, (MCCME, Moscow).

[2] Chase C., Serrano J., and Ramadge P.J., 1993, Periodicity and chaos from switched flow systems: contrasting examples of discretely controlled continuous systems. IEEE Trans. Automatic Control, $38,70-83$.

[3] Horn C., Ramadge P.J., 1997, A topological analysis of a family of dynamical systems with non-standard chaotic and periodic behavior. Int. J. Control, 67, 979-996.

[4] Katok A., Hasselblatt B., 1995, Introduction to the modern theory of dynamical systems (Cambridge Univ. Press).

[5] Katzorke I., Pikovsky A., 2000, Chaos and complexity in a simple model of production dynamics. Discrete Dynamics in Nature and Society, 5, 179-187.

[6] Peters K., Parlitz U., 2003, Hybrid systems forming strange billiards. Int. J. of Bifurcations and Chaos, 19, 2575-2588.

[7] Schurmann T., Hoffman I., 1995, The entropy of strange billiards inside n-simplexes. J. Phys. A 28, 5033-5039.

[8] Tsujii M., 2001, Absolutely continuous invariant measures for expanding piecewise linear maps. Invent. Math. 143:2, 349-373. 